\title{
Stem Cells from a Biological Perspective in Animals: A Review
}

\author{
M. A. Khan, Archana Jain, J. Shakkarpude* \\ Department of Veterinary Physiology, College of Veterinary Science \& A.H., Mhow, NDVSU (MP) 453446
}

\begin{abstract}
Cells are the smallest living units of living system. Stem cells are undifferentiated biological cells that can differentiate into specialized cells and can divide to produce more stem cells. Embryonic stem (ES) cells are the inner cell mass of a blastocyst, an earlystage embryo. (Thomson et al., 1998). ES cells are pluripotent and give rise during development to all derivatives of the three primary germ layers: ectoderm, endoderm and mesoderm. Adult stem cells, also called somatic stem cells, they are stem cells which maintain and repair the tissue in which they are found (Behrens et al., 2014). Pluripotent adult stem cells are rare and generally small in number, but they can be found in umbilical cord blood and other tissues. Embryonic stem cells can be grown relatively easily in culture. Adult stem cells are rare in mature tissues. Cell potency is a cell's ability to differentiate into other cell types. Stem cells resembling totipotent blastomeres from 2-cell stage embryos can arise spontaneously in the embryonic stem cell cultures. (Macfarlan et al., 2012). New research related to multipotent cells suggests that multipotent cells may be capable of conversion into unrelated cell types. In one case, fibroblasts were converted into functional neurons. Stem-cell therapy is the use of stem cells to treat or prevent a disease or condition.
\end{abstract}

Keywords - Stem cell, Types, Identification, Properties, Cell potency, Stem cell therapy, Medical use.

\section{INTRODUCTION}

Stem cells are a pervasive component of embryonic and foetal development, tissue maintenance and of regeneration and repair. Stem cells are potential source of new cells for the therapeutic regeneration of diseased or damaged tissue. Embryonic Stem cells are pluripotent and have retained their embryonic capacity to give rise to most, probably all, cell types. The potential advantage of ES cells is their ability to be isolated and grown in large numbers coupled with their ability to differentiate into any other cell of the body. Tissue transplantation carries with it the risk of immune reaction and rejection unless the donor cells are closely immunologically matched or (ideally) identical to those of the patient. One seductively enticing scenario would be to provide a source of ES cells, genetically identical to the patient from which that required specialised pre- cursor cells might be differentiated. This would involve "de-differentiation" of a cell from the patient to an ES state. The only method so far known to work in animal model experiments is nuclear transplantation into an oocyte and establishment of the ES cell line from the early embryo formed. Such somatic cell nuclear transfer (SCNT) technique could be used to generate a patient specific ES cell line. This scenario of generating an embryo by nuclear transfer of an adult cell nucleus into an enucleated oocyte, making an ES cell line and using this to generate cells which will repopulate tissues of an adult, has been fully demonstrated in a mouse model.

\section{Stem Cell}

Stem cells are undifferentiated biological cells that can differentiate into specialized cells and can divide (through mitosis) to produce more stem cells. They are found in multicellular organisms. In mammals, there are two broad types of stem cells: embryonic stem cells, which are isolated from the inner cell mass of blastocysts, and adult stem cells, which are found in various tissues. In adult organisms, stem cells and progenitor cells act as a repair system for the body, replenishing adult tissues. In a developing embryo, stem cells can differentiate into all the specialized cells ectoderm, endoderm and mesoderm but also maintain the normal turnover of regenerative organs, such as blood, skin, or intestinal tissues.

Stem cells are distinguished from other cell types by two important characteristics. First, they are unspecialized cells capable of renewing themselves through cell division, sometimes after long periods of inactivity. Second, under certain physiologic or experimental conditions, they can be induced to become tissue or organ-specific cells with special functions.

\section{History of stem cell}

In the mid 1800s it was discovered that cells were basically the building blocks of life and that some cells had the ability to produce other cells. In 1968, the first bone marrow transplant was performed to successfully treat two siblings with severe combined 
immunodeficiency. Other key events in stem cell research include:

1978: Stem cells were discovered in human cord blood

1981: First in vitro stem cell line developed from mice

1988: Embryonic stem cell lines created from a hamster

1995: First embryonic stem cell line derived from a primate

1997: Cloned lamb from stem cells

1997: Leukaemia origin found as haematopoietic stem cell, indicating possible proof of cancer stem cells.

\section{Properties of all stem cells}

Stem have three general properties:

They are capable of dividing and renewing themselves for long periods, they are unspecialized and they can give rise to specialized cell types.

\section{Embryonic stem cells}

Embryonic stem (ES) cells are the cells of the inner cell mass of a blastocyst, an early-stage embryo. Thomson et al., (1998). ES cells are pluripotent and give rise during development to all derivatives of the three primary germ layers: ectoderm, endoderm and mesoderm. During embryonic development these inner cell mass cells continuously divide and become more specialized.

\section{Embryonic stem cells grown in the laboratory}

Growing cells in the laboratory is known as cell culture. Embryonic stem cells are generated by transferring cells from a pre-implantation stage embryo into a plastic laboratory culture dish that contains a nutrient broth known as culture medium. The cells divide and spread over the surface of the dish. The inner surface of the culture dish is typically coated with mouse embryonic skin cells that have been treated so they will not divide. This coating layer of cells is called a feeder layer. The mouse cells in the bottom of the culture dish provide the cells a sticky surface to which they can attach. Also, the feeder cells release nutrients into the culture medium. Researchers have devised ways to grow embryonic stem cells without mouse feeder cells. This is a significant scientific advance because of the risk that viruses or other macromolecules in the mouse cells may be transmitted to the human cells.

The process of generating an embryonic stem cell line is somewhat inefficient, so lines are not produced each time cells from the pre-implantation-stage embryo are placed into a culture dish. However, if the plated cells survive, divide, and multiply enough to crowd the dish, they are removed gently and plated into several fresh culture dishes. The process of re-plating or sub-culturing the cells is repeated many times and for many months. Each cycle of sub-culturing the cells is referred to as a passage. Once the cell line is established, the original cells yield millions of embryonic stem cells. Embryonic stem cells that have proliferated in cell culture for six or more months without differentiating, are pluripotent, and appear genetically normal are referred to as an embryonic stem cell line. At any stage in the process, batches of cells can be frozen and shipped to other laboratories for further culture and experimentation.

\section{Laboratory tests are used to identify embryonic stem cells}

However, laboratories that grow human embryonic stem cell lines use several kinds of tests, including:

1. Growing and sub-culturing the stem cells for many months. This ensures that the cells are capable of long-term growth and self- renewal.

2. Using specific techniques to determine the presence of transcription factors that are typically produced by undifferentiated cells. Two of the most important transcription factors are Nanog and Oct 4.

3. Using specific techniques to determine the presence of particular cell surface markers that are typically produced by undifferentiated cells.

4. Examining the chromosomes under a microscope. This is a method to assess whether the chromosomes are damaged or if the number of chromosomes has changed. It does not detect genetic mutations in the cells.

5. Determining whether the cells can be re-grown, or sub-cultured, after freezing, thawing, and replating.

6. Testing whether the human embryonic stem cells are pluripotent by allowing the cells to differentiate spontaneously in cell culture, manipulating the cells so they will differentiate to form cells characteristic of the three germ layers and injecting the cells into a mouse with a suppressed immune system to test for the formation of a benign tumor called a teratoma.

\section{Adult stem cells}

Adult stem cells, also called somatic stem cells, they are stem cells which maintain and repair the tissue in which they are found. Pluripotent adult stem cells are rare and generally small in number, but they can be found in umbilical cord blood and other tissues. (Ratajczak et al., 2007). Bone marrow is a rich source of adult stem cells, which have been used in treating several conditions including liver cirrhosis, chronic limb ischemia and end stage heart failure.

Adult stem cells are also used in veterinary medicine to treat tendon and ligament injuries in horses. The history 
of research on adult stem cells began about 50 years ago. In the $1950 \mathrm{~s}$, researchers discovered that the bone marrow contains at least two kinds of stem cells. One population, called hematopoietic stem cells, forms all the types of blood cells in the body. A second population, called bone marrow stromal stem cells (also called mesenchymal stem cells, or skeletal stem cells by some) were discovered a few years later.

\section{Identification and function of stem cells}

Adult stem cells have been identified in many organs and tissues, including brain, bone marrow, peripheral blood, blood vessels, skeletal muscle, skin, teeth, heart, gut, liver, ovarian epithelium, and testis. They are thought to reside in a specific area of each tissue (called a "stem cell niche"). In many tissues, current evidence suggests that some types of stem cells are pericytes, cells that compose the outermost layer of small blood vessels. Stem cells may remain quiescent (non-dividing) for long periods of time until they are activated by a normal need for more cells to maintain tissues, or by disease or tissue injury.

\section{Tests are used to identify adult stem cells}

Scientists often use one or more of the following methods to identify adult stem cells:

(1) Label the cells in a living tissue with molecular markers and then determine the specialized cell types they generate.

(2) Remove the cells from a living animal, label them in cell culture, and transplant them back into another animal to determine whether the cells replace (or "repopulate") their tissue of origin.

\section{Adult stem cell differentiation}

Normal differentiation pathways of adult stem cells. In a living animal, adult stem cells are available to divide for a long period, when needed, and can give rise to mature cell types that have characteristic shapes and specialized structures and functions of a particular tissue.

1. Hematopoietic stem cells:-Hematopoietic stem cell give rise to all the types of blood cells.

2. Mesenchymal stem cells:-Mesenchymal stem cell have been reported to be present in many tissues.

3. Neural stem cells:-Neural stem cells in the brain give rise to its three major cell types: nerve cells (neurons).

4. Epithelial stem cells:-Epithelial stem cell in the lining of the digestive tract occur in deep crypts and give rise to several cell types: absorptive cells, goblet cells, Paneth cells, and enteroendocrine cells.
5. Skin stem cells:-Skin stem cells occur in the basal layer of the epidermis and at the base of hair follicles.

\section{Differences between embryonic and adult stem cells}

One major difference between adult and embryonic stem cells is their different abilities in the number and type of differentiated cell types they can become. Embryonic stem cells can become all cell types of the body because they are pluripotent. Adult stem cells are thought to be limited to differentiating into different cell types of their tissue of origin. Embryonic stem cells can be grown relatively easily in culture. Adult stem cells are rare in mature tissues, so isolating these cells from an adult tissue is challenging.

\section{Cell Potency}

Cell potency is a cell's ability to differentiate into other cell types. The more cell types a cell can differentiate into, the greater its potency. Potency is taken from the Latin term "potens" which means "having power."

\section{Totipotency}

Totipotency is the ability of a single cell to divide and produce all of the differentiated cells in an organism. Spores and zygotesare examples of totipotent cells. Toti comes from the Latin totus which means "entirely". Stem cells resembling totipotent blastomeres from 2-cell stage embryos can arise spontaneously in the embryonic stem cell cultures. (Macfarlan et al., 2012). and also can be induced to arise more frequently in vitro through down-regulation of the chromatin assembly activity of CAF-1.

\section{Pluripotency}

Pluripotency (from the Latin plurimus, meaning very many, and potens, meaning having power) refers to a stem cell that has the potential to differentiate into any of the three germ layers: endoderm, mesoderm or ectoderm and nervous system.

\section{Induced pluripotency}

Induced pluripotent stem cells, commonly abbreviated as iPS cells or iPSCs are a type of pluripotent stem cell artificially derived from a non-pluripotent cell, typically an adult somatic cell, by inducing a "forced" expression of certain genes and transcription factors. These transcription factors play a key role in determining the state of these cells and also highlight the fact that these somatic cells do preserve the same genetic information as early embryonic cells. (Stadtfeld and Hochedlinger 2010). The ability to induce cells into a pluripotent state was initially pioneered in 2006 using 
mouse fibroblasts and four transcription factors, Oct4, Sox2, Klf4 and c-Myc; this technique, called reprogramming, earned Shinya Yamanaka and John Gurdon the Nobel Prize in Physiology or Medicine 2012.

\section{Multipotency}

Multipotency describes progenitor cells which have the gene activation potential to differentiate into multiple, but limited cell types. New research related to multipotent cells suggests that multipotent cells may be capable of conversion into unrelated cell types. In one case, fibroblasts were converted into functional neurons. (Vierbuchen et al., 2010). Multipotent cells are found in many, but not all human cell types. Multipotent cells have been found in cord blood, (Yong and Theodore 2010). Adipose tissue, cardiac cells, bone marrow, and mesenchymal stem cells (MSCs) which are found in the third molar. Hematopoietic stem cells are an example of multipotency.

\section{Oligopotency}

Oligopotency is the ability of progenitor cells to differentiate into a few cell types. It is a degree of potency. Examples of oligopotent stem cells are the lymphoid or myeloid stem cells.

\section{Unipotency}

A unipotent cell is the concept that one stem cell has the capacity to differentiate into only one cell type.

\section{Stem cell therapy}

Stem-cell therapy is the use of stem cells to treat or prevent a disease or condition: Bone marrow transplant is the most widely used stem-cell therapy, but some therapies derived from umbilical cord blood are also in use. Research is underway to develop various sources for stem cells, and to apply stem-cell treatments for neurodegenerative diseases and conditions such as diabetes, heart disease, and other conditions.

\section{Medical uses}

For over 30 years, bone marrow has been used to treat cancer patients with conditions such as leukaemia and lymphoma; this is the only form of stem-cell therapy that is widely practiced. (Karanes et al., 2008). Another stem-cell therapy called Prochymal, was conditionally approved in Canada in 2012 for the management of acute graft vs host disease in children who are unresponsive to steroids. It is an allogenic stem therapy based on mesenchymal stem cells (MSCs) derived from the bone marrow of adult donors.

\section{Neuro-degeneration}

Research has been conducted on the effects of stem cells on animal models of brain degeneration, such as in Parkinson's, Amyotrophic lateral sclerosis, and Alzheimer's disease. There have been preliminary studies related to multiple sclerosis.

\section{Heart}

Possible mechanisms of recovery include:

Generation of heart muscle cells, stimulation of growth of new blood vessels to repopulate damaged heart tissue, secretion of growth factors and assistance via some other mechanism.

\section{Wound healing}

Stem cells can also be used to stimulate the growth of human tissues. In an adult, wounded tissue is most often replaced by scar tissue, which is characterized in the skin by disorganized collagen structure, loss of hair follicles and irregular vascular structure.

\section{Infertility}

Culture of human embryonic stem cells in mitotically inactivated porcine ovarian fibroblasts (POF) causes differentiation into germ cells (precursor cells of oocytes and spermatozoa), as evidenced by gene expression analysis. (Richards et al., 2008). The stem cell, therapy may also be used in the treatment of the following: Brain and spinal cord injury, Blood cell formation, Missing teeth, Cochlear hair cell re-growth, Blindness and vision impairment, Pancreatic beta cell, Orthopaedics etc.

\section{Veterinary medicine}

Research currently conducted on horses, dogs, and cats can benefit the development of stem cell treatments in veterinary medicine and can target a wide range of injuries and diseases such as myocardial infarction, stroke, tendon and ligament damage, osteoarthritis, osteoc hondrosis and muscular dystrophy both in large animals, as well as humans. (Murphy et al., 2003).

\section{Sources of stem cells}

Veterinary applications of stem cell therapy as a means of tissue regeneration have been largely shaped by research that began with the use of adult-derived mesenchymal stem cells to treat animals with injuries or defects affecting bone, cartilage, ligaments and/or tendons. There are two main categories of stem cells used for treatments: allogeneic stem cells derived from a genetically different donor within the same species and autologous mesenchymal stem cells, derived from the patient prior to use in various treatments. A third category, xenogenic stem cells, or stem cells derived from 
different species, are used primarily for research purposes, especially for human treatments.

\section{Stem cells and hard-tissue repair}

Because of the general positive healing capabilities of stem cells, they have gained interest for the treatment of cutaneous wounds. In one trial, stem cells were isolated from the Wharton's jelly of the umbilical cord. These cells were injected directly into the wounds. Within a week, full re-epithelialization of the wounds had occurred, compared to minor re-epithelialization in the control wounds. This showed the capabilities of mesenchymal stem cells in the repair of epidermal tissues.

\section{Stem cells and orthopaedic repairs}

Logous stem cell-based treatments for ligament injury, tendon injury, osteoarthritis, osteocho ndrosis, and sub-Auto-chondral bone cysts have been commercially available to practicing veterinarians to treat horses since 2003 in the United States and since 2006 in the United Kingdom. Autologous stem cell based treatments for tendon injury, ligament injury, and osteoarthritis in dogs have been available to veterinarians in the United States since 2005. Over 3000 privately owned horses and dogs have been treated with autologous adipose-derived stem cells. The efficacy of these treatments has been shown in double-blind clinical trials for dogs with osteoarthritis of the hip and elbow and horses with tendon damage. (Nixon et al., 2008).

\section{Tendon repair}

The embryonic stem cells were shown to have a better survival rate in the tendon as well as better migrating capabilities to reach all areas of damaged tendon. The overall repair quality was also higher, with better tendon architecture and collagen formed.

\section{Joint repair}

Horses and dogs are most frequently affected arthritis. Adipose-derived mesenchymal cells are currently the most often used because of the non-invasive harvesting. There has been a lot of success recently injecting mesenchymal stem cells directly into the joint. This is a recently developed, non-invasive technique developed for easier clinical use. Dogs receiving this treatment showed greater flexibility in their joints and less pain. (Guercio et al., 2012).

\section{Bone defect repair}

Stem cells have been used to treat degenerative bone diseases. The normally recommended treatment for dogs that have Legg Calve Perthes disease. Recently, mesenchymal stem cells have been injected directly in to the head of the femur, with success not only in bone regeneration, but also in pain reduction.

\section{Stem cells and muscle repairs}

Stem cells have successfully been used to ameliorate healing in the heart after myocardial infarction in dogs. Adipose and bone marrow derived stem cells were removed and induced to a cardiac cell fate before being injected into the heart. The heart was found to have improved contractility and a reduction in the damaged area four weeks after the stem cells were applied.

\section{Stem cells and nervous system repairs}

Spinal cord injuries are one of the most common traumas brought into veterinary hospitals. Mesenchymal stem cells that are induced to a neural cell fate are loaded on to a porous scaffold and are then implanted at the site of injury. The cells and scaffold secrete factors that counteract those secreted by scar forming cells and promote neural regeneration. Eight weeks later, dogs treated with stem cells showed immense improvement over those treated with conventional therapies. Dogs treated with stem cells were able to occasionally support their own weight, which has not been seen in dogs undergoing conventional therapies. (Sung Su Park et al., 2012).

\section{REFERENCES}

[1] Behrens ,A., van Deursen, J.M., Rudolph, K.L. and Schumacher, B. (2014). "Impact of genomic damage and ageing on stem cell function". Nature Cell Biology 16 (3): 201-207.

[2] Guercio, A., Di Marco, P., Casella, S., Cannella, V., Russotto, L., Purpari, G., Di Bella. and Piccione., G. (2012). "Production of canine mesenchymal stem cells from Adipose tissue and their application in dogs with chronic osteoarthritis of the humero-radial joints". Cell Biology International. 36 (2): 189-94.

[3] Karanes, C., Nelson, G.O., Chitphakdithai, P., Agura, E., Ballen, K.K., Bolan, C.D., Porter D.L., Uberti, J.P., King, R.J. and Confer, D.L. (2008). "Twenty years of unrelated donor hematopoietic cell transplantation for adult recipients facilitated by the National Marrow Donor Program". Biology of Blood and Marrow Transplantation. 14 (9): 8-15.

[4] Macfarlan. T.S., Gifford, W.D., Driscoll, S., Lettieri, K., Rowe, H.M., Bonanomi, D., Firth , A., Singer, O., Trono, D. and Pfaff, S.L. (2012). "Embryonic stem cell potency fluctuates with endogenous retrovirus activity". Nature. 487: 57-63.

[5] Murphy, J.M., Fink, D.J., Hunziker, E.B. and Barry, F.P. (2003). "Stem cell therapy in a caprine model of osteoarthritis". Arthritis Rheum. 48 (12): 3464-3474. 
[6] Nixon, A.J., Dahlgren, L.A., Haupt, J.L., Yeager, A.E. and Ward, D.L. (2008). "Effect of adiposederived nucleated cell fractions on tendon repair in horses with collagenase-induced tendinitis". American. Journal of Veterinary Research 69 (7): 928-937.

[7] Ratajczak, M.Z., Machalinski, B., Wojakowski, W., Ratajczak, J. and Kucia, M. (2007). "A hypothesis for an embryonic origin of pluripotent Oct-4(+) stem cells in adult bone marrow and other tissues". Leukemia. 21 (5): 860-867.

[8] Richards, M., Fong, C.Y. and Bongso, A (2008). "Comparative evaluation of different in vitro systems that stimulate germ cell differentiation in human embryonic stem cells". Fertility and Sterility. 93 (3): 986-994.

[9] Stadtfeld, M. and Hochedlinger, K. (2010). "Induced pluripotency: history, mechanisms, and applications". Genes \& Development. 24 (20): 2239 -2263 .

[10] Sung Su Park. (2012). "Functional recovery after spinal cord injury in dogs treated with a combination of Matrigel and neural-induced adipose-derived mesenchymal Stem cells". Cytotherapy. 14 (5): 584597.

[11] Thomson, J.A., Itskovitz-Eldor, J., Shapiro, S.S., Waknitz, M.A., Swiergiel, J.J., Marshall, V.S. and Jones J.M. (1998). "Blastocysts Embryonic Stem Cell Lines Derived from Human". Science. 282(5391): 1145-1147.

[12] Vierbuchen, T., Ostermeier, A., Pang, Z.P., Kokubu, Y., Südhof, T.C. and Wernig, M (2010)."Direct conversion of fibroblasts to functional neurons by defined factors". Nature. 463 (7284): 1035-1041.

[13] Yong Zhao and Theodore Mazzone (2010). "Human cord blood stem cells and the journey to a cure for type 1 diabetes". Autoimmunity Reviews. 10 (2): 103-107. 